\title{
Theoretical Interpretation of the Even Levels in the First Spectrum of Tungsten*
}

\author{
Y. Shadmi** and E. Caspi**
}

\begin{abstract}
(August 2, 1968)
The calculated even levels of $\mathrm{W}_{\mathrm{I}}$ up to the height of about $40,000 \mathrm{~cm}^{-1}$, which is the height of the ground level of the $d^{6}$ configuration, are reported. Fifty-seven observed levels are fitted to them, with a mean error of $100 \mathrm{~cm}^{-1} . L-S$ coupling and configuration assignments are usually meaningless because of a very strong spin-orbit interaction and configuration interaction. For every level, the largest squared $L-S$ coupling components of its eigenvector are reported, as well as observed and calculated $g$-values.
\end{abstract}

Key Words: Atomic spectrum, W I; energy levels, W I; spectrum of tungsten; tungsten; W I.

Laun has published a list of new levels belonging to The $\mathrm{W}_{\mathrm{I}}$ spectrum [1]. ${ }^{1}$ He was kind enough to send us his list of even levels prior to their publication. He thought it desirable to have theoretical names for these new levels, as well as for the previously observed levels already published in Moore's book [2]. For a detailed history of their investigation, the reader is referred to AEL [2].

About seven years ago, one of the authors of the present paper (Y.S.) performed theoretical calculations of the configurations of the type $(5 d+6 s)^{n}$ for all the first spectra of the platinum group. The interaction between the three above-mentioned configurations and the spin-orbit interaction, which are both very strong in the platinum group, were included in the Hamiltonian. In these calculations, the analogous interaction parameters of the various spectra, as well as the differences between the centers of the three configurations, were expressed by interpolation formulas. This enabled us to include in our calculations the $d^{n}$ configurations (which are the highest ones) within the spectra of Hf I, Ta I and WI, in which levels belonging to configurations of this type are not experimentally known. For a more detailed description of the above-mentioned approximations and of the interpolative method, the reader is referred to analogous papers on the iron and palladium groups $[3,4,5]$.

During the past several years, we tried to improve the above-mentioned calculations on the first spectra of the platinum group by adding to their Hamiltonians

*An invited paper. This paper was partially supported by the National Bureau of Stand ards, Wa hington, D.

**Present address: The Hebrew University of Jerusalem.

Figures in brackets indicate the literature references at the end of this paper.

${ }^{2}$ We were glad to hear that Dr. Laun, to whom we had sent a preprint of our paper prior to its publication, has now decided to exclude these two levels from his new list of observed levels. various effective-interactions which had been successfully used in the iron group. It turned out, that in the platinum group, these new corrections did not improve the approximation.

In order to investigate the validity and interpretation of the new observed levels of W I, we simply used for the interaction parameters the interpolative values which we had obtained in our above mentioned previous calculations, and diagonalized the numerical energy matrix of W I obtained in this way. We used the derivatives of its renewed diagonalization ("Diag. l") for least-squares calculations in which we tried to fit all the observed levels with the calculated ones. Since the $d^{6}$ configuration is completely unknown, we determined its interaction parameters by forcing them into a linear progression with the analogous parameters of the $d^{4} s^{2}$ and $d^{5} s$ configurations. The difference between the additive parameters of $d^{6}$ and $d^{5} \mathrm{~s}\left(\mathrm{~A}-\mathrm{A}^{\prime}\right)$, which was extrapolated from the first spectra on the right-hand side of $\mathrm{W}_{\mathrm{I}}$, was fixed. The results of our calculations are as follows: Out of 38 even levels of W I lower than $40,000 \mathrm{~cm}^{-1}$ reported in AEL [2], we were able to fit 37 to the calculated ones. Out of the 24 levels now reported by Laun, we could fit 23 levels to our calculated ones. Thus the total number of observed levels fitted to the calculated ones is 60 . The level 22852.84 with $J=5$ reported in AEL and the level 30155.90 with $J=1$, now reported by Laun cannot be fitted to any calculated level. ${ }^{2}$ Three observed levels can be fitted to calculated ones, only with rather large deviations. These are the level 27670.48 with $J=1$, and the levels 28204.20 and 30374.20 , both with $J=2$. For details, the reader is referred to table 2 . In the first least-squares calculation ("L.S. la"), in which the three above mentioned levels were included, the mean error was $\pm 143 \mathrm{~cm}^{-1}$. In the second least- 
squares calculation ("L.S. 1b"), where these levels were not included, the mean error was reduced to $\pm 102 \mathrm{~cm}^{-1}$. Unfortunately, our present calculation is not good enough to enable us to make a final decision regarding the reality of these three observed levels. It is well known that in the first spectra of the platinum group, there are more even configurations which are not much higher than the $d^{n}$ configurations, which can perturb the relatively higher levels of the configurations $d^{n-1} s$. In the case of $\mathrm{W}_{\mathrm{I}}$, the additional perturbations are probably due to configurations having one $7 s$ electron, and perhaps configurations having one $6 d$ or two $6 p$ electrons. Thus, we believe that for some of the levels whose heights approach $30,000 \mathrm{~cm}^{-1}$, and, of course, for many higher ones, the predictions of our present approximation may be inaccurate. In order to achieve convergence, the parameters of L.S. lb were used for an additional diagonalization, which was followed by a least-squares calculation ("L.S. 2"). Also in L.S. 2, the three above mentioned problematic levels were not included, and the mean error was $\pm 100 \mathrm{~cm}^{-1}$.

The results of the present calculation are reported in two tables. In table 1 , we give the values obtained for the various interaction parameters. The symbols used for these parameters are the usual ones (see, for example, references [3, 4, 5]). Analogous parameters of the configurations $d^{4} s^{2}, d^{5} s$ and $d^{6}$, are designated by the same letter double primed, single primed, and unprimed, respectively. The values given in the column "Diag. 1" are the interpolated values used in the first diagonalization. It is worthwhile noticing that the values obtained for the parameters in the least-squares calculations, and which were determined by the observed material of $\mathrm{W}_{\mathrm{I}}$, assumed numerical
TABLE 1. W I $(5 d+6 s)^{6}$-Parameters of the various stages of the calculation

\begin{tabular}{|c|c|c|c|c|}
\hline & Diag. 1 & L.S. la & L.S. $1 \mathrm{~b}$ & L.S. 2 \\
\hline $\begin{array}{l}\mathrm{A}^{\prime \prime} \\
\mathrm{A}^{\prime}-\mathrm{A}^{\prime \prime} \\
\mathrm{A}-\mathrm{A}^{\prime} \\
\mathrm{B}^{\prime \prime} \\
\mathrm{B}^{\prime} \\
\mathrm{B} \\
\mathrm{C}^{\prime \prime} \\
\mathrm{C}^{\prime} \\
\mathrm{C} \\
\mathrm{G} \\
\mathrm{H} \\
\alpha \\
\zeta^{\prime \prime} \\
\zeta^{\prime} \\
\zeta\end{array}$ & $\begin{array}{r}17210 \\
15430 \\
14310 \\
485 \\
440 \\
395 \\
1843 \\
1735 \\
1627 \\
2650 \\
475 \\
39 \\
2080 \\
1875 \\
1670\end{array}$ & $\begin{array}{c}17442 \pm 395 \\
15361 \pm 451 \\
\text { Fixed } \\
499 \pm 9 \\
436 \pm 10 \\
\text { Prog. } \\
1867 \pm 30 \\
1736 \pm 30 \\
\text { Prog. } \\
2747 \pm 30 \\
483 \pm 7 \\
38 \pm 3 \\
2114 \pm 29 \\
1853 \pm 46 \\
\text { Prog. }\end{array}$ & $\begin{array}{c}17186 \pm 291 \\
16096 \pm 345 \\
\text { Fixed } \\
490 \pm 7 \\
451 \pm 8 \\
\text { Prog. } \\
1878 \pm 21 \\
1710 \pm 22 \\
\text { Prog. } \\
2731 \pm 21 \\
476 \pm 6 \\
39 \pm 2 \\
2108 \pm 21 \\
1889 \pm 35 \\
\text { Prog. }\end{array}$ & $\begin{array}{c}17152 \pm 286 \\
16190 \pm 340 \\
\text { Fixed } \\
489 \pm 7 \\
451 \pm 7 \\
\text { Prog. } \\
1880 \pm 21 \\
1707 \pm 20 \\
\text { Prog. } \\
2743 \pm 24 \\
475 \pm 6 \\
39 \pm 2 \\
2104 \pm 20 \\
1888 \pm 34 \\
\text { Prog. }\end{array}$ \\
\hline Mean error & & \pm 143 & \pm 102 & \pm 100 \\
\hline
\end{tabular}

Notes:

1. The symbols for the parameters as given in the first column are the usual ones. For their detailed definition, see, for example, Refs. 3, 4, and 5.

2. The abbreviation "Prog." denotes that the value of that parameter was determined by forcing it into a linear progression with the analogous parameters of the two lower configurations.

values rather close to the interpolative ones (which were determined by the observed material of all the first spectra of the Pt group).

In table 2, observed and calculated energy levels and $g$ values are given. The calculated energy levels are those obtained in L.S. 2. The three levels not included in this L.S. calculation are given in brackets, and so are their deviations. For $J=0,1,2,3$ and 4, we reported only those calculated levels which are lower than the ${ }^{5} \mathrm{D}$ of $d^{6}$ and this ${ }^{5} \mathrm{D}$ itself. For higher $J$ numbers, we reported the levels up to the height of $40,000 \mathrm{~cm}^{-1}$.

In the column "assignment," we gave the con-

TABLE 2. W I-Observed and calculated energy levels

\begin{tabular}{|c|c|c|c|c|c|c|}
\hline Assignment & $J$ & Obs. & Calc. & $\mathrm{O}-\mathrm{C}$ & Obs. $g$ & Calc. $g$ \\
\hline $69 \% d^{4} s^{25} \mathrm{D}$. & 0 & 0.00 & -99 & 99 & & \\
\hline$d^{4} s^{2}\left[47 \%^{3} \mathrm{P}+24 \%^{5} \mathrm{D}+19 \%^{1} \mathrm{~S}\right]$ & 0 & 9528.07 & 9480 & 48 & & \\
\hline 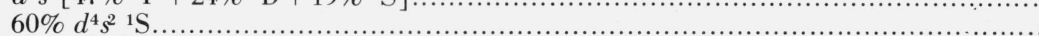 & 0 & 20174.20 & 20086 & 88 & & \\
\hline 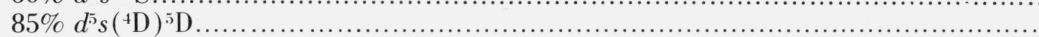 & 0 & 22773.78 & 22872 & -98 & & \\
\hline$\left[55 \% d^{5}\left({ }^{4} \mathrm{P}\right) s+31 \% d^{4} s^{2}\right]^{3} \mathrm{P}$. & 0 & & 29106 & & & \\
\hline$\left[43 \% d^{4} s^{2}+32 \% d^{5} s+13 \% d^{6}\right]^{3} \mathrm{P}$. & 0 & & 35204 & & & \\
\hline$\left[34 \% d^{5} s+24 \% d^{4} s^{2}\right]\left[42 \%{ }^{1} \mathrm{~S}+17 \%{ }^{3} \mathrm{P}\right]+23 \% d^{6} \mathrm{D} \ldots$ & 0 & & 40939 & & & \\
\hline 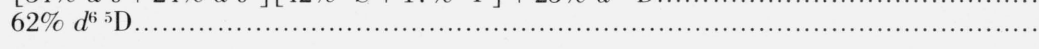 & 0 & & 41888 & & & \\
\hline $84 \% d^{4} s^{25} \mathrm{D} .$. & 1 & 1670.30 & 1697 & -27 & 1.51 & 1.498 \\
\hline $67 \% d^{4} s^{23} \mathrm{P} \ldots$ & 1 & 13307.06 & 13405 & -98 & 1.32 & 1.303 \\
\hline$\left[46 \% d^{4} s^{2}+21 \% d^{5} s\right]^{3} \mathrm{D}$ & 1 & 18082.80 & 18095 & -12 & 0.7 & 0.784 \\
\hline $65 \% d^{5} s\left({ }^{4} \mathrm{P}\right)^{5} \mathrm{P} \ldots \ldots \ldots \ldots$. & 1 & 20427.81 & 20407 & 21 & 2.1 & 2.100 \\
\hline$d^{5} s\left[56 \%\left({ }^{4} \mathrm{D}\right)^{5} \mathrm{D}+28 \%\left({ }^{+} \mathrm{P}\right)^{5} \mathrm{P}\right]$ & 1 & 23455.02 & 23541 & -86 & & 1.720 \\
\hline $74 \% d^{5} s\left({ }^{4} \mathrm{~F}\right)^{5} \mathrm{~F} \ldots \ldots \ldots \ldots \ldots \ldots \ldots \ldots \ldots \ldots \ldots \ldots \ldots$ & 1 & $(27670.48)$ & 28123 & $(-453)$ & & 0.210 \\
\hline$\left[41 \% d^{5}\left({ }^{4} \mathrm{P}\right) s+37 \% d^{4} s^{2}\right]^{3} \mathrm{P} \ldots \ldots$ & 1 & 28720.88 & 28877 & -156 & & 1.395 \\
\hline$d^{5} s\left[56 \%\left({ }^{2} \mathrm{D}\right)^{3} \mathrm{D}+18 \%\left({ }^{4} \mathrm{D}\right)^{3} \mathrm{D}\right] \ldots$ & 1 & 32378.40 & 32443 & -65 & & 0.539 \\
\hline $43 \% d^{5} s\left({ }^{4} \mathrm{D}\right)^{3} \mathrm{D}+25 \% d^{4} s^{23} \mathrm{P} \ldots \ldots$ & 1 & & 32639 & & & 0.871 \\
\hline $34 \% d^{5} s\left({ }^{2} \mathrm{D}\right)^{3} \mathrm{D}+\left[28 \% d^{5}\left({ }^{4} \mathrm{P}\right) s+17 \% d^{4} s^{2}\right]^{3} \mathrm{P}$. & 1 & & 35756 & & & 1.058 \\
\hline 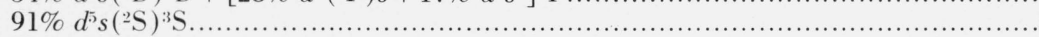 & 1 & & 37538 & & & 1.973 \\
\hline $85 \% d^{65} \mathrm{D} \ldots \ldots \ldots \ldots$ & 1 & & 41269 & & & 1.488 \\
\hline 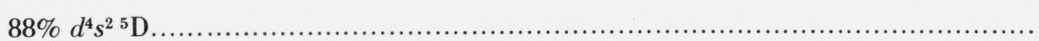 & 2 & 3325.53 & 3382 & -57 & 1.48 & 1.485 \\
\hline 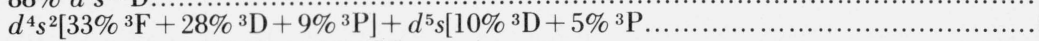 & 2 & 13777.70 & 13708 & 70 & 1.09 & 1.042 \\
\hline 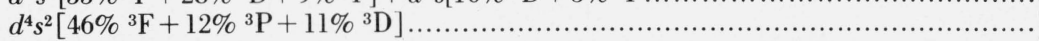 & 2 & 14976.21 & 15058 & -82 & 1.06 & 0.982 \\
\hline
\end{tabular}




\begin{tabular}{|c|c|c|c|c|c|c|}
\hline Assignment & $J$ & Obs. & Calc. & $\mathrm{O}-\mathrm{C}$ & Obs. $g$ & Calc. $g$ \\
\hline 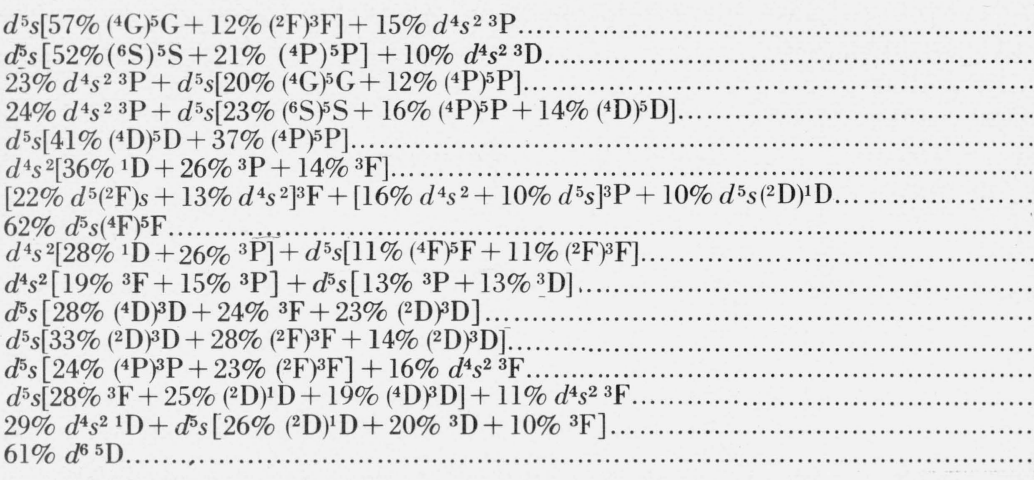 & $\begin{array}{l}2 \\
2 \\
2 \\
2 \\
2 \\
2 \\
2 \\
2 \\
2 \\
2 \\
2 \\
2 \\
2 \\
2 \\
2 \\
2 \\
2\end{array}$ & $\begin{array}{l}18116.84 \\
18280.48 \\
19253.58 \\
20983.06 \\
23982.80 \\
24789.66 \\
26861.64 \\
(28204.20) \\
28898.96 \\
(30374.20)\end{array}$ & $\begin{array}{l}18240 \\
18385 \\
19283 \\
21031 \\
24028 \\
24799 \\
26844 \\
28576 \\
28790 \\
29840 \\
31037 \\
34341 \\
35128 \\
35753 \\
37463 \\
40340\end{array}$ & $\begin{array}{r}-123 \\
-105 \\
-30 \\
-48 \\
-45 \\
-9 \\
18 \\
(-372) \\
109 \\
(534)\end{array}$ & $\begin{array}{l}1.08 \\
1.43 \\
1.18\end{array}$ & $\begin{array}{l}0.724 \\
1.759 \\
1.214 \\
1.582 \\
1.562 \\
1.123 \\
1.029 \\
1.025 \\
1.129 \\
1.127 \\
1.057 \\
0.963 \\
1.079 \\
0.919 \\
1.033 \\
1.334\end{array}$ \\
\hline 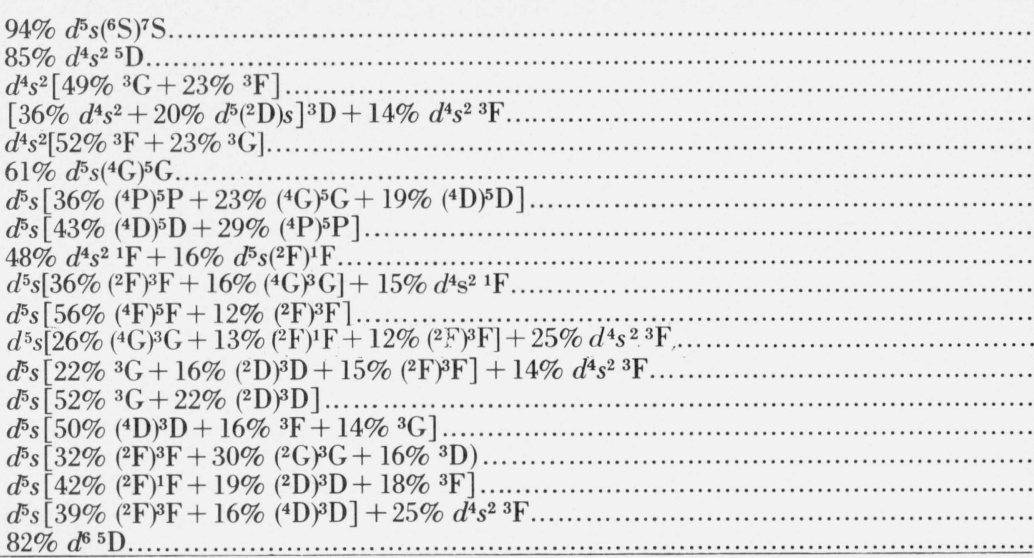 & $\begin{array}{l}3 \\
3 \\
3 \\
3 \\
3 \\
3 \\
3 \\
3 \\
3 \\
3 \\
3 \\
3 \\
3 \\
3 \\
3 \\
3 \\
3 \\
3 \\
3 \\
3 \\
3 \\
3\end{array}$ & $\begin{array}{r}2951.29 \\
4830.00 \\
13348.54 \\
15459.99 \\
17701.14 \\
18974.47 \\
19827.67 \\
23930.08 \\
24610.90 \\
28291.88 \\
28347.60\end{array}$ & $\begin{array}{r}2833 \\
4836 \\
13424 \\
15405 \\
17736 \\
18994 \\
19759 \\
23870 \\
24741 \\
28196 \\
28454 \\
29409 \\
31996 \\
32821 \\
33675 \\
34566 \\
37143 \\
38562 \\
39911\end{array}$ & $\begin{array}{r}118 \\
-6 \\
-76 \\
55 \\
-35 \\
-20 \\
69 \\
60 \\
-130 \\
96 \\
-106\end{array}$ & $\begin{array}{l}1.98 \\
1.50 \\
0.92 \\
1.17 \\
1.02 \\
1.06 \\
1.28\end{array}$ & $\begin{array}{l}1.978 \\
1.475 \\
0.942 \\
1.256 \\
1.020 \\
1.089 \\
1.400 \\
1.488 \\
1.034 \\
1.060 \\
1.173 \\
1.035 \\
1.060 \\
1.008 \\
1.178 \\
1.072 \\
1.174 \\
1.452\end{array}$ \\
\hline 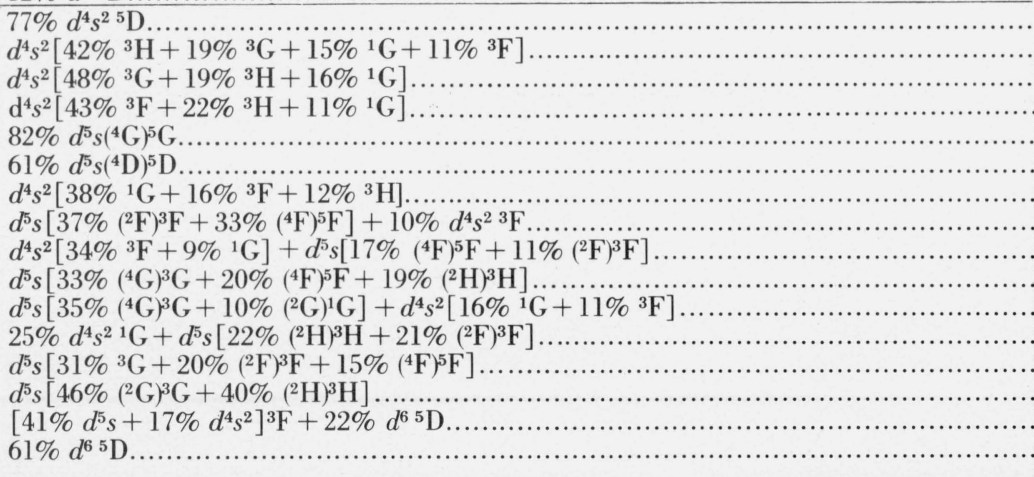 & $\begin{array}{l}4 \\
4 \\
4 \\
4 \\
4 \\
4 \\
4 \\
4 \\
4 \\
4 \\
4 \\
4 \\
4 \\
4 \\
4 \\
4 \\
4\end{array}$ & $\begin{array}{r}6219.33 \\
12161.95 \\
16431.28 \\
17107.02 \\
19256.23 \\
22476.68 \\
22852.80 \\
27213.82 \\
29479.32 \\
29853.66 \\
32135.94 \\
\\
34302.04\end{array}$ & $\begin{array}{l}6105 \\
12162 \\
16567 \\
17043 \\
19275 \\
22442 \\
22826 \\
27114 \\
29453 \\
2913 \\
31902 \\
33421 \\
34159 \\
35219 \\
37030 \\
38229\end{array}$ & $\begin{array}{r}114 \\
0 \\
-136 \\
64 \\
-19 \\
35 \\
27 \\
100 \\
26 \\
41 \\
234 \\
\\
143\end{array}$ & $\begin{array}{l}1.49 \\
0.99 \\
1.02 \\
1.19 \\
1.20 \\
1.48\end{array}$ & $\begin{array}{l}1.452 \\
0.995 \\
1.010 \\
1.117 \\
1.179 \\
1.414 \\
1.077 \\
1.241 \\
1.230 \\
1.081 \\
1.070 \\
1.036 \\
1.147 \\
0.970 \\
1.284 \\
1.405\end{array}$ \\
\hline 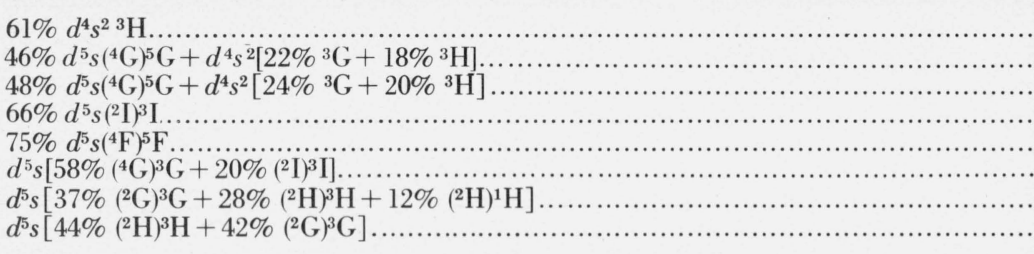 & $\begin{array}{l}5 \\
5 \\
5 \\
5 \\
5 \\
5 \\
5 \\
5\end{array}$ & $\begin{array}{l}15069.94 \\
19535.04 \\
19826.04 \\
27849.80 \\
28233.44 \\
31389.08 \\
33291.80\end{array}$ & $\begin{array}{l}15124 \\
19391 \\
20035 \\
27755 \\
28273 \\
31256 \\
33352 \\
36364\end{array}$ & $\begin{array}{r}-54 \\
144 \\
-209 \\
95 \\
-40 \\
133 \\
-60\end{array}$ & $\begin{array}{l}1.05 \\
1.21 \\
1.20\end{array}$ & $\begin{array}{l}1.097 \\
1.203 \\
1.198 \\
0.933 \\
1.338 \\
1.114 \\
1.146 \\
1.117\end{array}$ \\
\hline 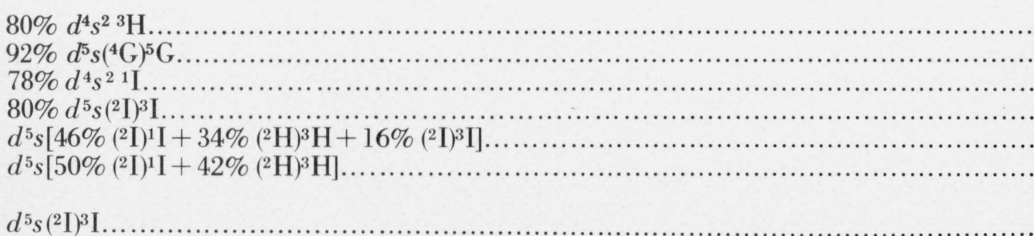 & $\begin{array}{l}6 \\
6 \\
6 \\
6 \\
6 \\
6\end{array}$ & $\begin{array}{l}17008.50 \\
19648.56 \\
23484.78 \\
28392.72\end{array}$ & $\begin{array}{l}16983 \\
19599 \\
23424 \\
28482 \\
33276 \\
37206\end{array}$ & $\begin{array}{r}26 \\
50 \\
61 \\
-89\end{array}$ & $\begin{array}{l}1.4 \\
1.32\end{array}$ & $\begin{array}{l}1.138 \\
1.318 \\
1.033 \\
1.054 \\
1.069 \\
1.078\end{array}$ \\
\hline
\end{tabular}


figuration and term-assignment of each level, when these quantum numbers are meaningful. Unfortunately, in the WI spectrum, we have a case of typical intermediate-coupling and strong configuration interaction as well. In many cases, the eigenvectors do not have one dominant $L-S$ coupling component. In these cases, the assignment column gives the largest squared components of the eigenvector. For some levels belonging to the $d^{5} s$ configuration, we report the term assignment without specifying the parent-term. This is done in cases where various parent-terms are mixed.

It is worthwhile mentioning that, in most cases, the assignments given by Laporte and Mack [6] to the even levels up to the approximate height of 20,000 $\mathrm{cm}^{-1}$ coincide with the $L-S$ assignments of the largest squared components of the corresponding calculated eigenvector as reported in table 2 .

The second author of the present paper (E. C.) is now doing research on configuration-interaction in the platinum group spectra. We hope that in these calculations we shall be able to take into account more interacting configurations. Only then, shall we be able to make a final decision as to the reality of the observed levels which seem doubtful according to the present calculation. Then, we shall probably also have reliable predictions for higher even levels.

\section{References}

[1] Laun, Donald D., and Corliss, C. H., J. Res. NBS 72A (Phys. and Chem.) No. 6609 (1968).

[2] Moore, C. E., “Atomic Energy Levels", NBS Circular 467, Vol. III (1958).

[3] Racah, G., and Shadmi, Y., Bull. Res. Council Israel 8F, No. 1, $15,(1959)$.

[4] Shadmi, Y., Bull. Res. Council Israel, 9F, No. 4, 141 (1961).

[5] Shadmi, Y., J. Res. NBS $\mathbf{7 0 A}$ (Phys. and Chem.) No. 5, 435 (1966).

[6] Laporte, O., and Mack, J. E., Phys. Rev. 63, 246 (1943).

(Paper 72A6-523) 